\title{
Effect of the Basin Shape on Formation of the Black Sea Circulation
}

\author{
A.A. Pavlushin, N.B. Shapiro, E.N. Mikhailova
}

\author{
Marine Hydrophysical Institute, Russian Academy of Sciences, Sevastopol, \\ Russian Federation \\ e-mail: pavlushin@mhi-ras.ru
}

\begin{abstract}
The results of numerical modeling of the wind circulation in the basins of various configurations, with horizontal bottom and with no account of the $\beta$-effect are represented. The present paper is a continuation of a series of experiments on numerical modeling the processes of formation of the Black Sea hydrophysical fields using a two-layer eddy-resolving model. Stationary cyclonic wind is used as an external forcing. Enegy sink takes place due to the bottom friction and the horizontal turbulent friction. The used in the model empirical coefficients in the equation terms describing friction are chosen in such a way that the circulation resulting from the simulations should correspond qualitatively to the one observed in the Black Sea. It is shown that at the prolonged forcing of the cyclonic wind in the stretched basins, the large-scale circulation arising due to vorticity of the wind tangential tention is divided into a few sub-basin gyres tending to take a shape of circles inscribed in the basin geometry. The number of such gyres depends on the ratio between the basin length and width: the more stretched the pool, the larger the number of the induced vortices. The processes of the current fields' formation in the basin are also affected by the coastline features. The experiments confirmed that formation of two separate cyclonic vortices in the Black Sea known as "Knipovich Glasses" is conditioned by the stretched shape of the basin and the influence of the Anatolian peninsula extension.
\end{abstract}

Keywords: the Black Sea, eddy-resolving model, numerical experiment, hydrodynamic instability of currents, basin shape.

DOI: 10.22449/1573-160X-2016-2-3-13

(C) 2016, A.A. Pavlushin, N.B. Shapiro, E.N. Mikhailova

(C) 2016, Physical Oceanography

Introduction. The aim of this paper is to study the influence of the shape and characteristics of the basin coastline on the circulation in the Black Sea. Particularly, causes of the formation of large-scale circulation feature, known as "Knippovich Glasses", is of some interest.

To study this issue the two-layer eddy-resolving model [1] was used. This model is similar to the Holland - Lin model for ocean circulation [2]. Circulation in the basin is generated by the wind. The energy flux is performed due to horizontal turbulent viscosity and bottom friction. The following model equations are the "primitive" equations of motion and continuity, integrated in within each layer:

$$
\begin{gathered}
\left(U_{1}\right)_{t}+\left(u_{1} U_{1}\right)_{x}+\left(v_{1} U_{1}\right)_{y}-f V_{1}=g h_{1} \zeta_{x}+\tau^{x}-R_{a}^{x}+A_{l} \nabla\left(h_{1} \nabla u_{1}\right), \\
\left(V_{1}\right)_{t}+\left(u_{1} V_{1}\right)_{x}+\left(v_{1} V_{1}\right)_{y}+f U_{1}=g h_{1} \zeta_{y}+\tau^{y}-R_{a}^{y}+A_{l} \nabla\left(h_{1} \nabla v_{1}\right), \\
\left(U_{2}\right)_{t}+\left(u_{2} U_{2}\right)_{x}+\left(v_{2} U_{2}\right)_{y}-f V_{2}=g h_{2} \zeta_{x}+g^{\prime} h_{2}\left(h_{1}\right)_{x}+R_{a}^{x}-R_{b}^{x}+A_{l} \nabla\left(h_{2} \nabla u_{2}\right), \\
\left(V_{2}\right)_{t}+\left(u_{2} V_{2}\right)_{x}+\left(v_{2} V_{2}\right)_{y}+f U_{2}=g h_{2} \zeta_{y}+g^{\prime} h_{2}\left(h_{1}\right)_{y}+R_{a}^{y}-R_{b}^{y}+A_{l} \nabla\left(h_{2} \nabla v_{2}\right), \\
\left(h_{1}\right)_{t}+\left(U_{1}\right)_{x}+\left(V_{1}\right)_{y}=0, \\
\left(h_{2}\right)_{t}+\left(U_{2}\right)_{x}+\left(V_{2}\right)_{y}=0,
\end{gathered}
$$


where indices 1 and 2 indicate the top and bottom layer, respectively; the subindices $x, y, t$ mean the differentiation; $u_{i}, v_{i}$ are the horizontal components of the velocity of currents in the $i$-layer; $h_{1}, h_{2}$ are the thickness of the layers; $U_{i}=u_{i} h_{i}$ and $V_{i}=v_{i} h_{i}$ are components of the flows; $R_{a}^{x}=r_{a}\left(u_{1}-u_{2}\right)$ and $R_{a}^{y}=r_{a}\left(v_{1}-v_{2}\right)$ are the friction force components between the layers; $R_{b}^{X}=r_{b} u_{2}$ and $R_{b}^{y}=r_{b} v_{2}$ are components of the bottom friction force; $r_{a}$ and $r_{b}$ are constant coefficients; $f=f_{0}+\beta y$ is the Coriolis parameter, $f_{0}=10^{-4} 1 / \mathrm{s}$, $\beta=2 \cdot 10^{-13} 1 /(\mathrm{cm} \cdot \mathrm{s}) ; \quad g=980 \quad \mathrm{~g} \cdot \mathrm{cm} / \mathrm{s}^{2} \quad$ is acceleration of gravity; $g^{\prime}=g\left(\rho_{2}-\rho_{1}\right) / \rho_{2} ; \tau^{x}$ and $\tau^{y}$ are tangential wind stress components; $A_{l}$ is the horizontal turbulent viscosity coefficient.

To close the equations the integral continuity equation in the rigid-lid approximation permitting to introduce the function of the current $\psi$ for total fluxes is used:

$$
U_{1}+U_{2}=-\psi_{y}, \quad V_{1}+V_{2}=\psi_{x} .
$$

At the side boundaries adhesion conditions are used. River runoff in the sea and water exchange through the straits is not taken into account. Initially, the fluid is at rest, the interface and the surface of the sea horizontal.

Finite-difference scheme, based on box method with B-grid (according to Arakawa terminology), two-layer time integration scheme, implicit friction approximation on the surface of the division and at the bottom and also the Coriolis force, is used for numerical approximation. Advective terms in the equations of continuity and motion equations are approximated by the approximation by the first accuracy order scheme (be the directed differences) in the equations of continuity; in the equations of motion for the approximation of the advective terms the Lax - Wendroff scheme of the second accuracy order is applied. Accounting of the scheme viscosity in the equations of continuity ensures the stability of the numerical scheme and the positive definiteness of the layer thickness.

In the numerical experiments described in [1], the calculations were carried out on a $4 \times 3 \mathrm{~km}$ rectangular grid with 0.1 hour time step. Tangential wind stress $\tau$ (Fig. 1) was set stationary with the constant vorticity $\left(0,5 \cdot 10^{-7} \mathrm{~N} / \mathrm{m}^{3}\right)$. Values $\tau^{x}$ and $\tau^{y}$ in the integration domain boundaries are $1 \mathrm{~cm}^{2} / \mathrm{s}^{2}$, the maximum values $\tau$ above the sea matched the $8 \mathrm{~m} / \mathrm{s}$ wind speed. The actual configuration of the Black Sea basin was also under consideration, but the bottom topography, $\beta$-effect and friction at the layer interface had not taken into account. It was shown that as a result of wind effect the circulation appeared at sea eventually formed sub-basin consisting of two cyclones. On its periphery the mesoscale anticyclones owing to baroclinic instability appeared. Fig. 2 shows the instantaneous (Fig. 2a, b) and time-averaged (Figure 2, $b, d$ ) thickness (Fig. 2, $a, b$ ) fields $h_{1}$ and fields of currents (Fig. 2, $c, d$ ) in the upper layer. Note that mesoscale anticyclones do not appear in the time-averaged circulation.

In the lower layer the circulation similar to one in the upper layer appeared [1]. Velocity of currents in the lower layer was lower than in the upper one (areaaveraged values were $\sim 17 \mathrm{~cm} / \mathrm{s}$, the maximum ones - up to $45 \mathrm{~cm} / \mathrm{s}$ ). In the upper layer the maximum velocities reached $100 \mathrm{~cm} / \mathrm{s}$, the mean ones were about $30 \mathrm{~cm} / \mathrm{s}$. 


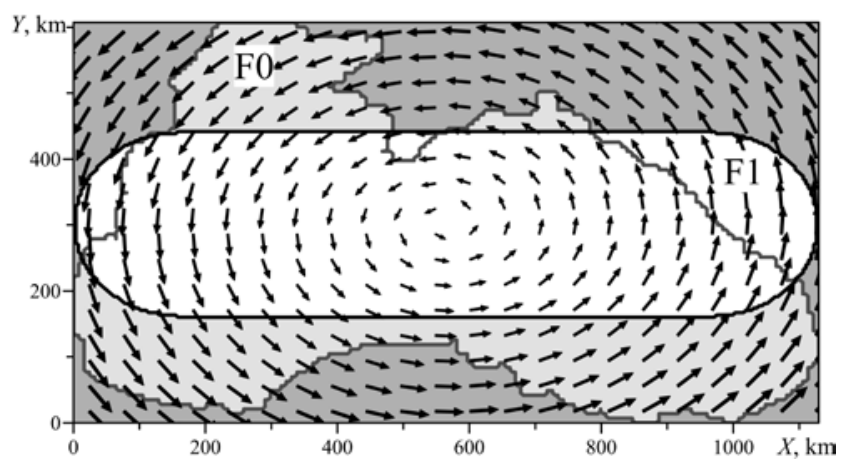

Fig. 1. Tangential wind stress field and shapes of the basins in the experiments F0 and F1
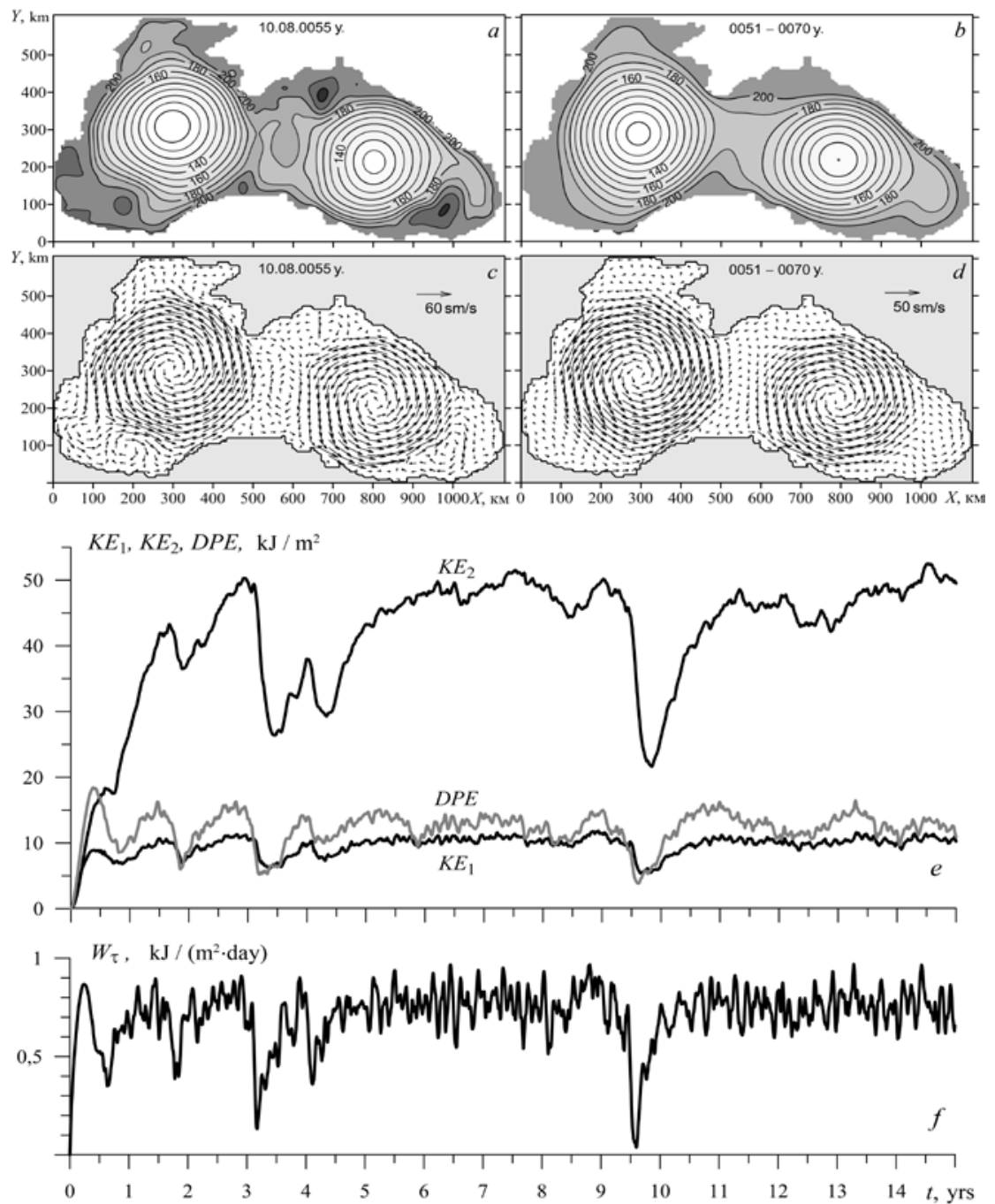

Fig. 2. Results of the calculations in the experiment F0 with the real Black Sea configuration: instantaneous ( $a$ ) and 20 year averaged $(b)$ thickness of the upper layer $h_{1}, \mathrm{~m}$; instantaneous $(c)$ and 20 year averaged $(d)$ field of currents in the upper layer; area-averaged components of the energy $(e)$ and action of the wind tangential stress $W_{\tau}(f)$

PHYSICAL OCEANOGRAPHY NO. 2 (2016) 
A comparison of the fields of different characteristics revealed that in the upper layer the thickness distribution was well-correlated with the field of current, and the integral function of the electric current reflected the currents in the lower layer. Therefore, for visualization of the circulation processes instead of vector fields of currents the distribution $h_{1}$ for the upper layer and $\psi$ - for the lower one can be used.

In addition to the instantaneous and mean fields of characteristics the analysis the time change graphs of the area-averaged kinetic and available potential energy (Fig. 2,e) was performed, defining the values of the aforementioned energy types as follows:

$$
K E_{1}=\left\langle\rho_{1} h_{1}\left(u_{1}^{2}+v_{1}^{2}\right) / 2\right\rangle, \quad K E_{2}=\left\langle\rho_{2} h_{2}\left(u_{2}^{2}+v_{2}^{2}\right) / 2\right\rangle, \quad D P E=\left\langle\rho_{1} g^{\prime}\left(h_{1}-h_{0}\right)^{2} / 2\right\rangle,
$$

where $K E_{1}$ is kinetic energy of the upper layer; $K E_{2}$ is kinetic energy of the lower layer; $D P E$ is the available potential energy; $\rho_{1}, \rho_{2}$ is water density in the upper and lower layer. Angle brackets mean area averaging

In these graphs the energy fluctuations with varying frequency and amplitude were observed. They are related to the action of the wind tangential stress (Fig. 2, $f)$, defined as $W_{\tau}=\left\langle\rho_{1}\left(u_{1} \tau^{x}+v_{1} \tau^{y}\right)\right\rangle$. Under the stationary external influence such fluctuations have self-oscillating character [3].

Numerical experiments. To make the further description more convenient, we introduced the following designations for each experiment. The aforementioned experiment with the Black Sea real configuration was called F0. The new one with the stretched shape of the basin (Fig. 1) was called F1. It was carried out to compare its results with the results of the F0 experiment. All parameters of the model, except the basin shape, were left unchanged, namely: the basin depth $H=$ $=2,200 \mathrm{~m}$; the upper layer thickness at the reference time was set equal to $175 \mathrm{~m}$; horizontal eddy viscosity coefficient $A_{l}=10^{5} \mathrm{~cm}^{2} / \mathrm{s}$; bottom friction coefficient $r_{b}=$ $=0,007 \mathrm{~cm} / \mathrm{s}$; friction between the layers was not taken into consideration $r_{a}=0$ ). The same wind field was used (Fig. 1).

In the F1 experiment in three years model enters a stable self-oscillating mode with the oscillation period $\sim 50$ days. This is clearly seen in the graphs of energy components and tangential wind stress action (Fig. 3, $a, b$ ) which differ from those in the graphs of the F0 experiment (Fig. 2, $e, f$ ).

Fig. 3 clearly shows three consequent periods of the circulation formation spin-up, adaptation and statistical equilibrium. The duration of the spin-up period is $\sim 5$ months. The values of $D P E$ and $K E_{1}$ are increasing monotonically and reach their maximum at the end of the first period, $K E_{2}$ is also growing, but less intensively than $K E_{1}$ and $D P E$. Energy growth takes place owing to the increase of $W_{\tau}$. At the end of the spin-up period $W_{\tau}$ reaches its maximum. This period ends when, due to the hydrodynamic instability of large-scale currents, the opposite wind vorticity mesoscale eddies begin to form, resulting in changing of the field of currents and decreasing of $W_{\tau}$. 

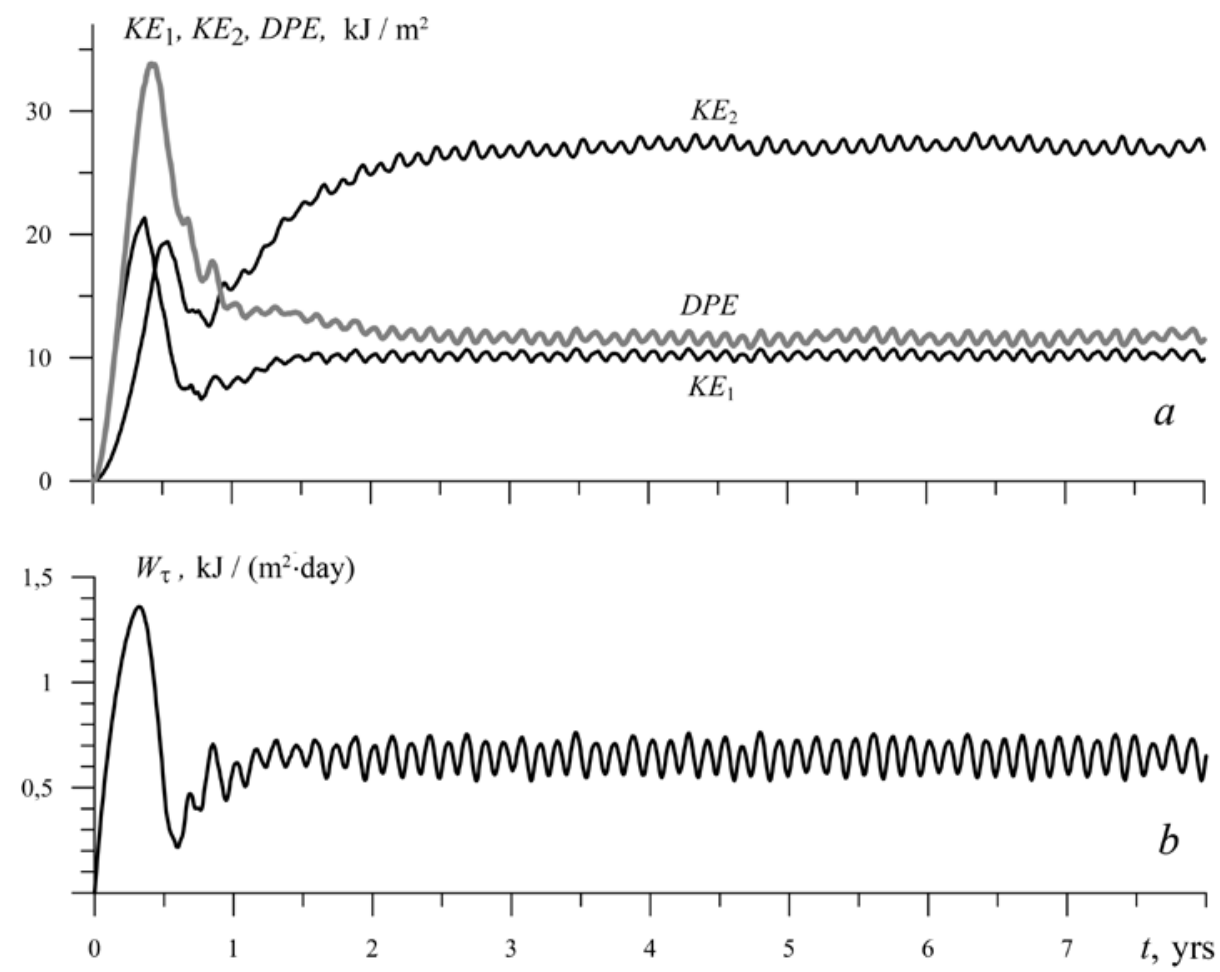

Fig. 3. Time-change graphs of the components of the energy $K E_{1}, K E_{2}, D P E(a)$ and tangential wind stress action $W_{\tau}(b)$ in the F1 experiment

At the beginning of the adaptation period due to a decrease of $W_{\tau}$ the values of $D P E$ and $K E_{1}$ reduce. As for $K E_{2}$, it continues its initial growth during several months, but then also begins to decrease (Fig. 3,a). The increase of $K E_{2}$ at the start of the period is obviously due to the fact that the available potential energy becomes part of the kinetic energy of the lower layer. Having reached its minimum approximately in the seventh month, $W_{\tau}$ begins to increase again, and, for this reason, the input of energy in a system making increase $K E_{1}$ and $K E_{2}$ grow, starts. The oscillations, which are then manifested in the behavior of energy components, are superimposed on the growth of $W_{\tau}$. The end of the adaptation period is the time when all the energy characteristics of the model enter a statistically equilibrium almost periodic mode, which persists until the end of the calculations. In this experiment, the adaptation period ends approximately at the end of the third year.

In the statistical equilibrium mode, the $D P E, K E_{1}, K E_{2}$ and $W_{\tau}$ graphs have a pronounced oscillatory character, with the amplitudes and frequency of oscillations being nearly stationary as opposed to the F0 experiment with the real configuration of the Black Sea (Fig. 2, $e, f$ ). The real shape of the basin and uneven coastline cause the formation of a greater number of mesoscale eddies which, interacting with each other and with large-scale currents, distort the "ideal" pattern observed in the F1 experiment. Mean $K E_{1}$ and $D P E$ values in these experiments are approximately equal. $K E_{2}$ in the $\mathrm{F} 0$ experiment is greater than in the F1. 
If we compare the oscillations in the graphs in Fig. 3, we can see that $W_{\tau}, D P E$ and $K E_{1}$ are in the same phase, and $K E_{2}$ - in the opposite phase. Most likely, this is the result of the energy transfer features in the lower layer when $D P E$ becomes $K E_{2}$ by means of the mesoscale eddies formed due to hydrodynamic instability currents. The cause of the oscillations is the energy inflow change in the system at the expense of $W_{\tau}$ (Fig. 3, $b$ ), which depends on the relative position of vector fields of wind and currents in the upper layer. This process has a self-oscillating character.

In Fig. 4, demonstrating several consequent distributions of the upper layer thickness within one period of the oscillations, you can see the way how the intensity of cyclones and anticyclones change. At the time when $W_{\tau}$ has its maximum (Fig. 4, $a$ ), there are three of the cyclones in the basin, the most intense of which is located in the middle. The anticyclonic vortices moving from the coast to the center are formed near the northern and southern boundaries of the basin between the cyclones (Fig. 4, b). At the minimum $W_{\tau}$ values the anticyclonic vortices between the cyclones are located closer to the middle axis of the basin (Fig. 4, c). Reducing of the energy inflow in the system leads to the weakening and disappearance of anticyclonic vortices (Fig. $4, d$ ). Then the $W_{\tau}$ growth starts again and oscillating system returns to the initial state (Fig. 4, $e$ ).

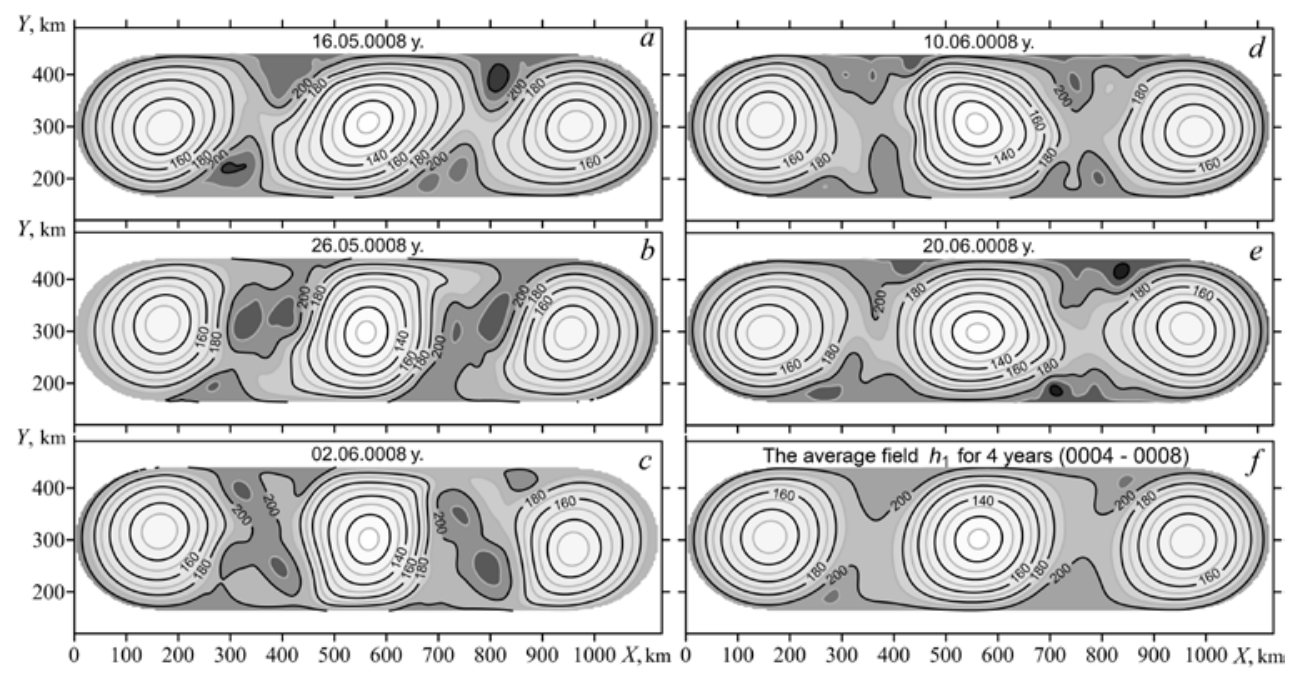

Fig. 4. Upper layer thickness $h_{1}(\mathrm{~m})$ for different time points in the F1 experiment (regions with larger thickness values are indicated with a darker color)

Fig. 4, $e$ shows the distribution of the upper layer thickness $h_{1}$, averaged over the entire period of statistical equilibrium. It is evident that in the averaged field, there are three sub-basin cyclonic gyres, and mesoscale anticyclonic vortices are virtually absent.

Thus, in the F1 experiment, as well as in the F0 experiment with the real configuration, the large-scale cyclonic circulation was divided into several gyres. Each gyre stroke to gain a rounded shape which was the maximum possible in the given boundaries of the basin. The difference lies in the number of cyclonic vortices formed, and also in the number and location of the areas of formation of mesoscale anticyclones. 
Note that in the F1experiment the known feature of the large-scale circulation in the Black Sea, namely the presence of two separate cyclonic vortices in the Black Sea (known as "Knipovich Glasses" [4]), was not revealed. In the subsequent experiments, there was an attempt to get the two-centered cyclonic circulation in the stretched basin by changing the ratio of its axes and configuration. Shapes of the basins, which were used in the calculations, are shown in Fig. 5. All other parameters of the model and the wind were set similarly to the F1 experiment.
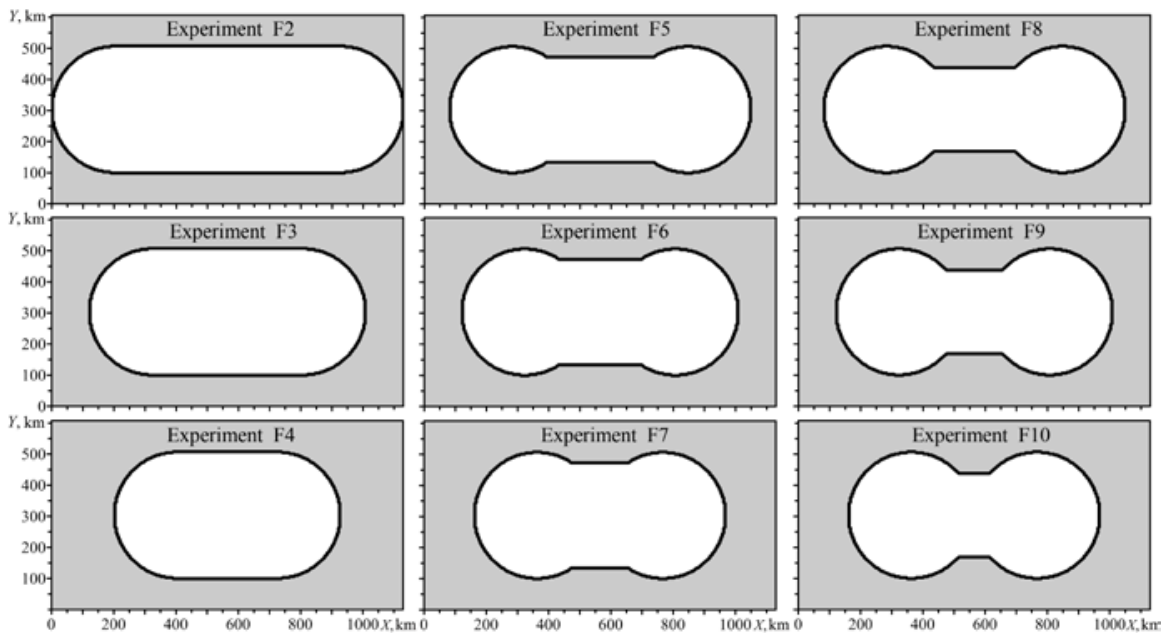

Fig. 5. Shapes of the basins in the experiments F2 - F10

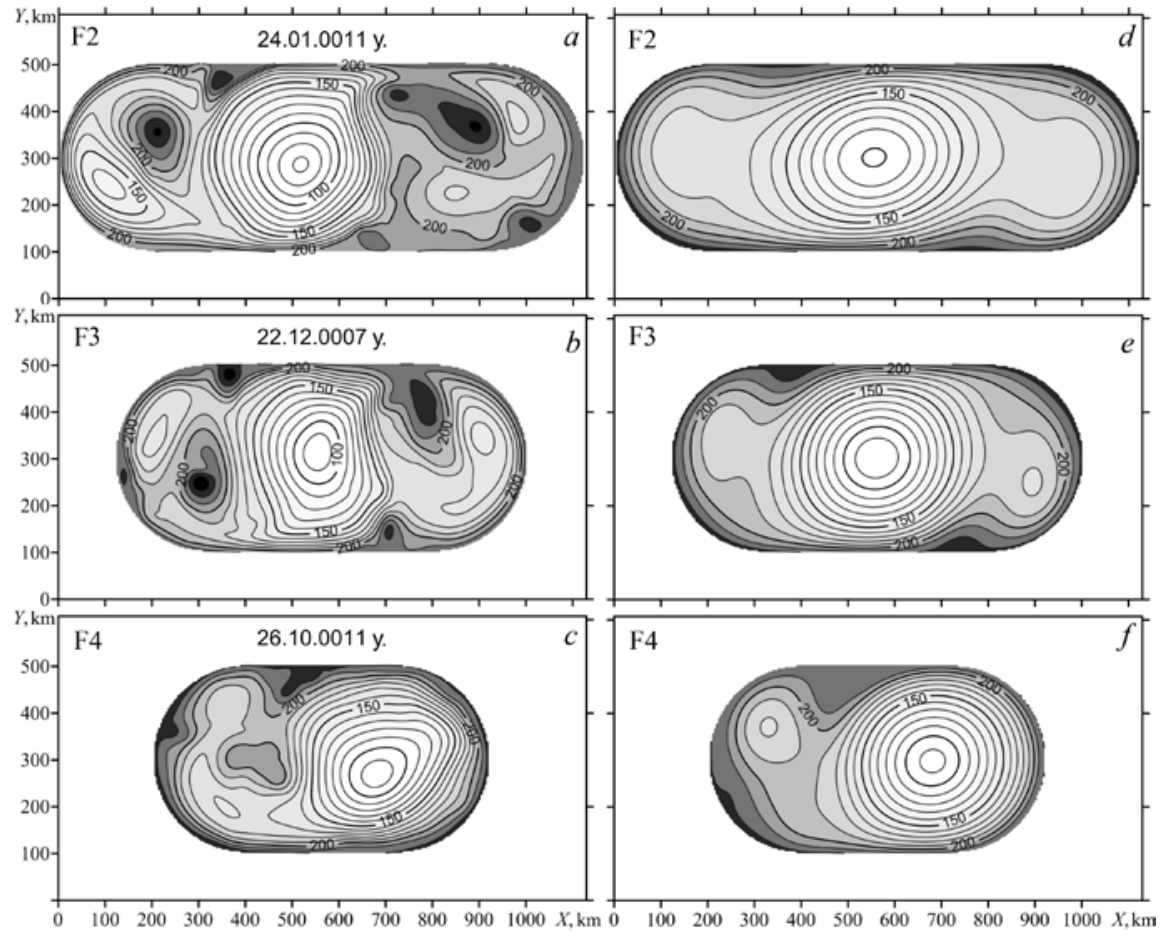

Fig. 6. Instantaneous $(a, b, c)$ and averaged $(d, e, f)$ fields $h_{1}(\mathrm{~m})$ in the $\mathrm{F} 2, \mathrm{~F} 3$ and $\mathrm{F} 4$ experiments

PHYSICAL OCEANOGRAPHY NO. 2 (2016) 
In the F1 experiment with the circulation, consisting of three cyclonic gyres, the ratio of the basin length $L$ to its width $B$ was about 3.6. In the F2, F3 and F4 experiments the $L / B$ ratio was 2.6; 2.1 and 1.6 respectively. Fig. $6, a$, d shows the results of the F2 experiment. There is a large cyclonic gyre in the middle of the basin and unstable cyclones on the left and right of it (Fig. 6, a). These cyclones didn't manifest in the averaged field (Fig. 6, d). Also, the circulation, consisting of two cyclones, could not be obtained in the F3 and F4 experiments (Fig. 6, $b, e$ and 6, $c, f$ respectively).

In the further experiments, we used a dumbbell-shaped form of the basin. In the F5, F6 and F7 experiments the width of the middle part ("handle") was set greater than in the F8, F9 and F10 experiments. Also, such basins differed from each other in length. Note that the two-centered circulation was formed only in the F5 experiment in the most stretched basin (Fig. 7, $a, d$ ). Two large-scale cyclonic vortices were developed in the eastern and western circular areas. Anticyclones were observed in the instantaneous fields in the region between the cyclones in the basin center - the "dumbbell handle" (Fig. 7, a). In the F5 experiment averaged field $h_{1}$ there was a zone of cyclonic vorticity in the "dumbbell handle" is (Fig. 7, $d$ ).

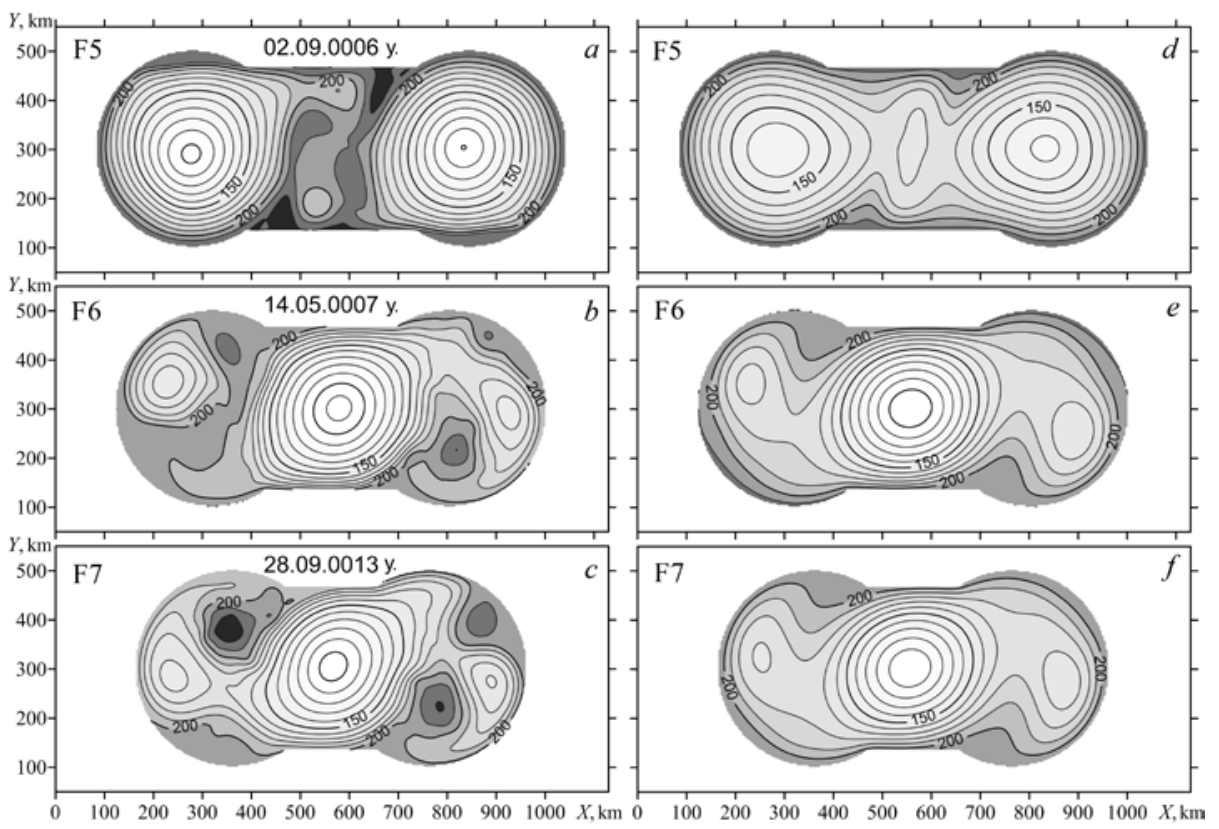

Fig. 7. Instantaneous $(a, b, c)$ and averaged $(d, e, f)$ fields $h_{1}(\mathrm{~m})$ in the F5, F6 and F7 experiments

In two other experiments, F6 and F7 (Fig. 7, $b, e, c$ and $f$ ) in the basin there was only one cyclone formed in the center. Mesoscale eddies were observed on the left and right of it.

In the F8, F9 and F10 experiments (dumbbell-shaped basins with a narrow "handle") the division of the large-scale circulation in the two gyres occurred in all three cases with instantaneous fields slightly different from the averaged ones in the long basins (Fig. 8, $a$ and $d$ ): the large-scale cyclonic vortices were located on the circular regions and the anticyclonic ones - only in the "handle". 
It's turned out that the essential condition for the development of two stable large-scale eddies in the Black Sea is the existence of a narrowed region in its central part. This assertion is true under the assumptions made of the horizontal bottom and a constant Coriolis parameter, but as is well-known, the large area of western part of the sea is occupied by the north-western shelf with the depths of less than $200 \mathrm{~m}$ (Fig. 9). If only the deep-water part of the Black Sea is considered (e.g. limited by $500 \mathrm{~m}$ isobath), it will, in rough approximation, be crescent-shaped.

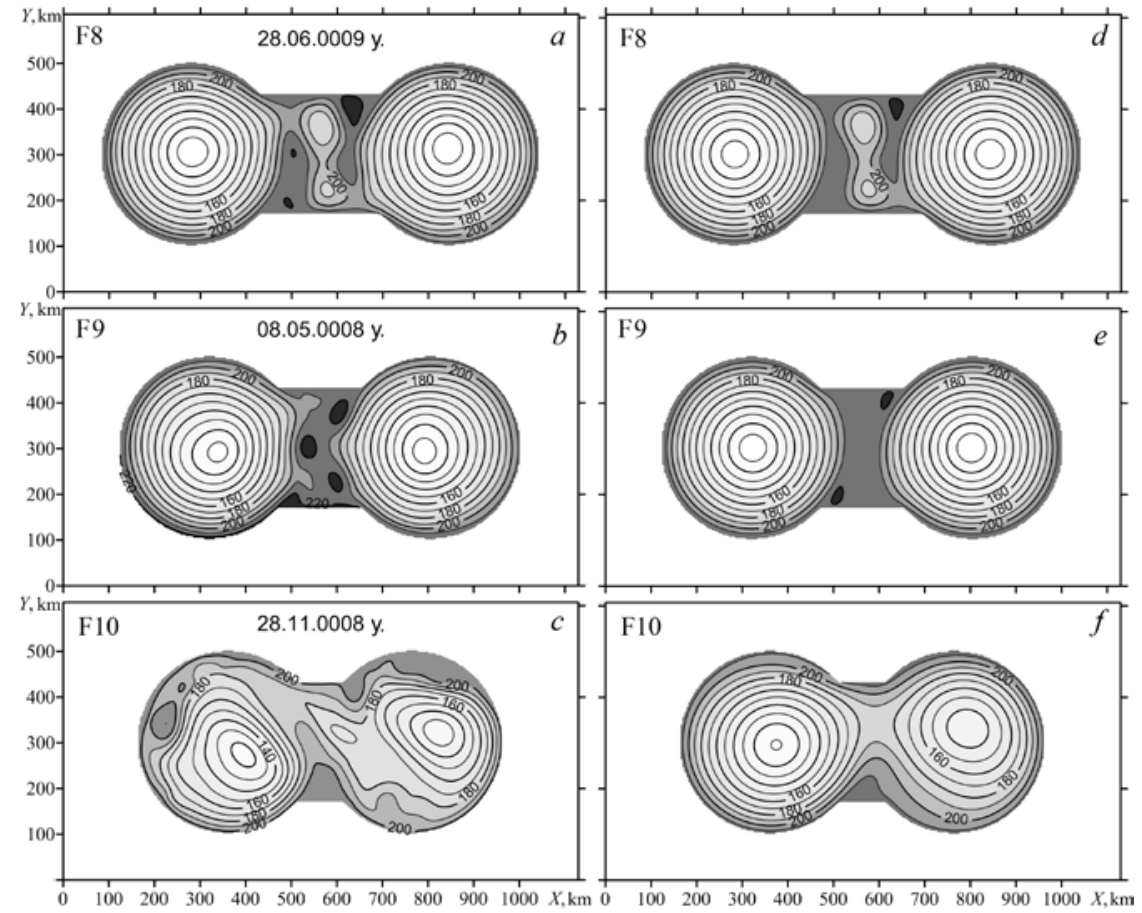

Fig. 8. Instantaneous $(a, b, c)$ and averaged $(d, e, f)$ fields $h_{1}(\mathrm{~m})$ in the F8, F9 and F10 experiments

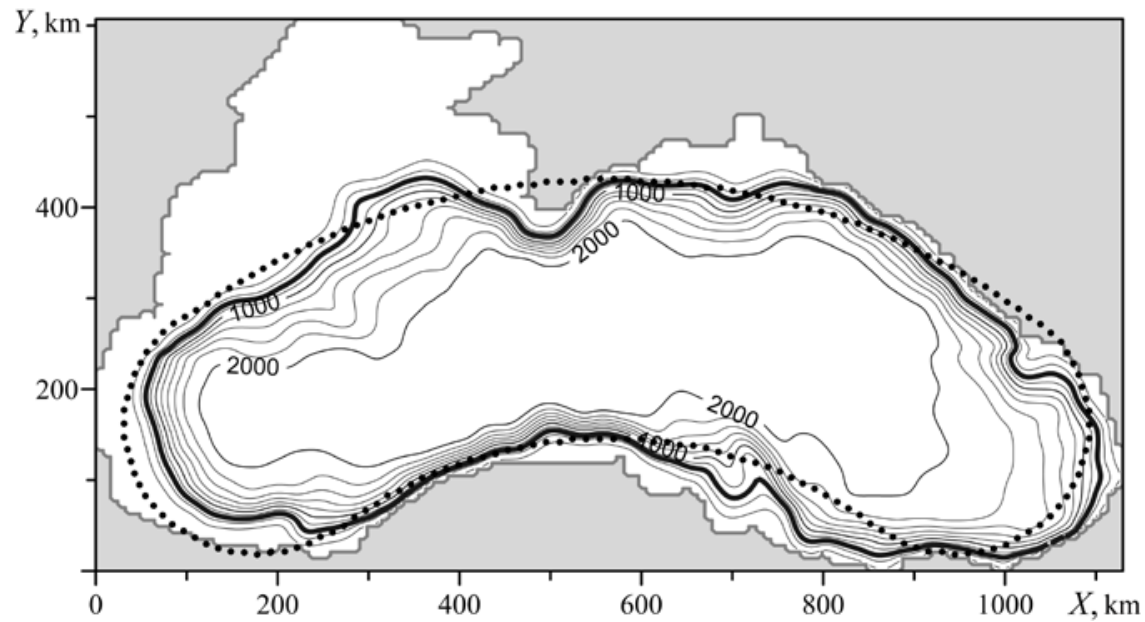

Fig. 9. The Black Sea bathymetrical map (m). The dotted line shows the shape of the basin used in the F11 experiment

PHYSICAL OCEANOGRAPHY NO. 2 (2016) 
The additional calculations were also performed. See their results in Fig. 10. In the F11 and F12 experiments curved shape of the basin. The two large-scale cyclonic gyres, one in the western and eastern parts, were formed in each of them under the influence of a stationary cyclonic wind within two year period. In the F11 experiment (Fig. 10, $c$ ) due to the greater length of the basin in the averaged fields there are two additional low intensity cyclones off the eastern boundary and in the central part of the basin. In the F12 experiment with the smaller basin length the weak cyclonic vortex can be found only in the east (Fig. 10, d).

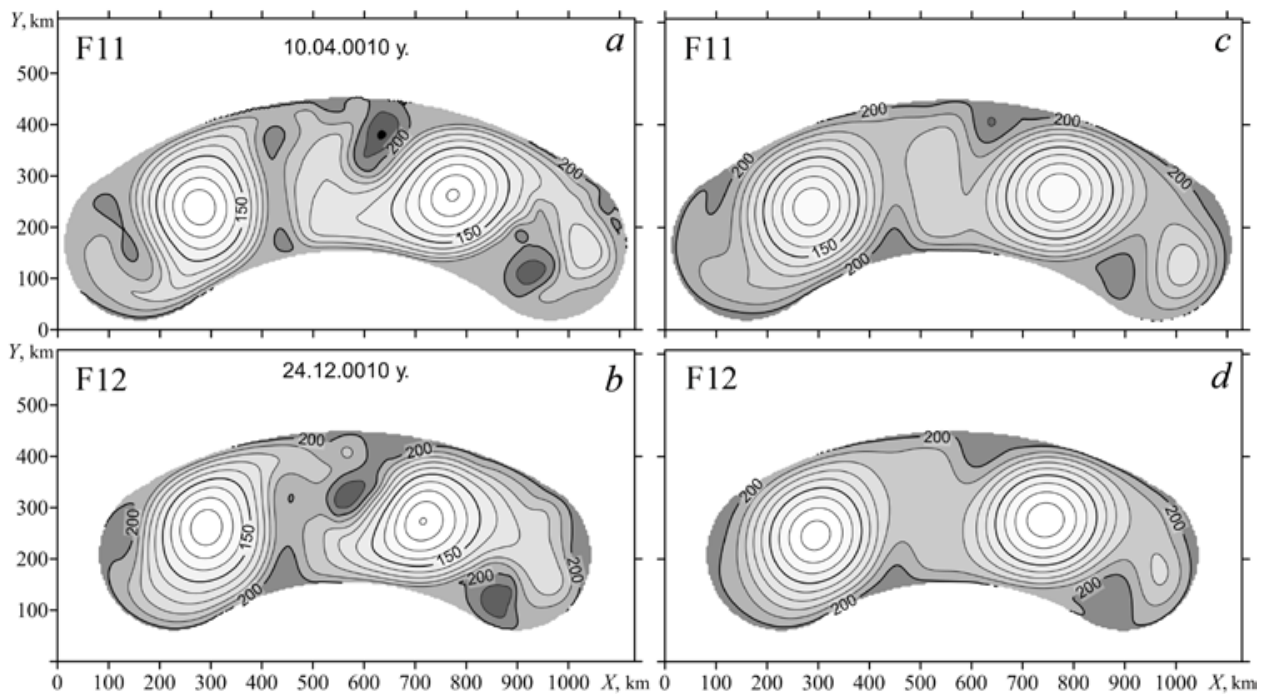

Fig. 10. Instantaneous $(a, b)$ and averaged $(c, d)$ fields $h_{1}(\mathrm{~m})$ in the F11 and F12 experiments
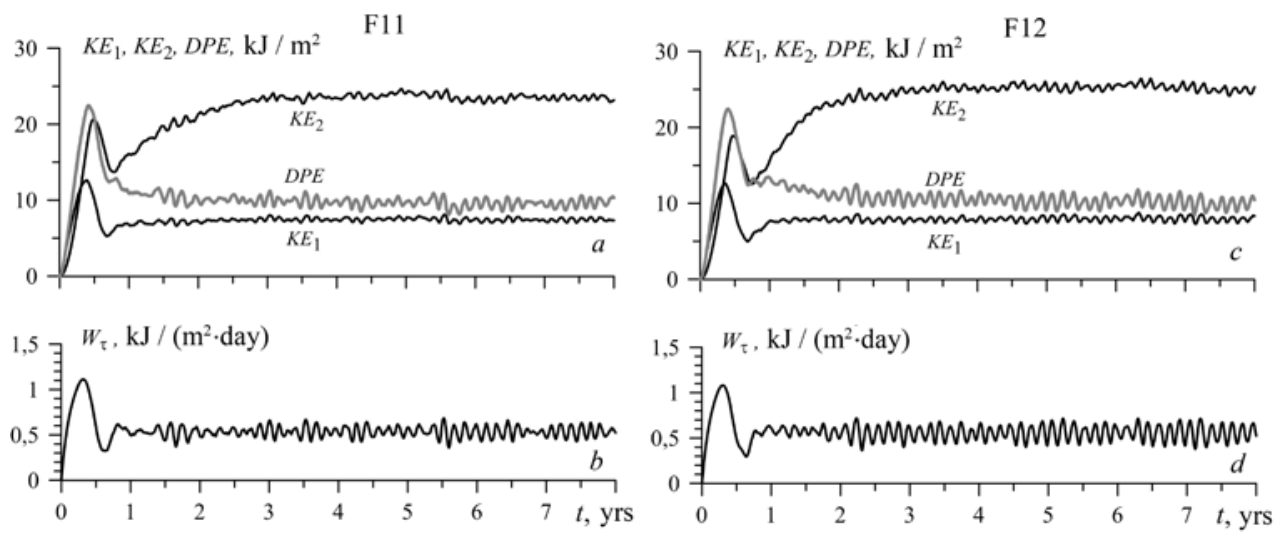

Fig. 11. Graphs of time changes in the $K E_{1}, K E_{2}$ and $D P E(a, c)$ energy components and tangential wind stress action $W_{\tau}(b, d)$

Graphs of energy and action of the tangential wind stress obtained in the F11 and F12 experiments are shown in Fig. 11. Generally, they are similar to the graphs in the F1 experiment (Fig. 3), but, unlike them, they are less stable (especially in the F11 experiment). 
Conclusions. Analyzing the results of the experiments performed, it can be said that the shape of the basin has a significant influence on the circulation development in the sea. In particular, the duration of the spin-up and adaptation periods, the number and the spatial distribution of large-scale eddies forming the main circulation depend on the basin shape.

In the stretched basins the large-scale circulation arising due to the wind vorticity is divided into a few sub-basin gyres tending to take a shape of circles inscribed in the basin geometry. The number of such gyres depends on the ratio between the basin length and width $(L / B)$. In the aforementioned experiments the circulation, consisting of two stationary cyclones could not be obtained by the $L / B$ variations in the stretched basin. In the basin when $L / B<2.8$ there is only one stable cyclonic gyre, located in the central part. When $L / B>3.5$ three quasistationary sub-basin gyres are formed.

The presence of significant coastal projections (peninsula) in the stretched basins also leads to the large-scale circulation division in the individual vortices. On the other hand, these basins can be considered to consist of several parts interconnected by straits. In this case, the circulation division into separate branches seems quite natural.

If we evaluate the results obtained relating to the Black Sea, it may be noted that the basins with the stretched curved shape (the F11 and F12 experiments) possess the most suitable parameters for it.

Therefore, the experiments confirmed the formation of two separate cyclonic vortices in the Black Sea known as "Knipovich Glasses" to be conditioned by the stretched shape of the basin and the influence of the Anatolian peninsula extension.

It should be emphasized that the described results were obtained for the basins with horizontal bottom and at the constant Coriolis parameter. Taking in account the bottom topography and the $\beta$-effect also plays a significant role in the circulation formation both in the Black Sea, and, for example, in the Sea of Japan [5, 6], as was stated by the reader of the present work (we bring him our sincere gratitude).

Acknowledgements. The work was carried out under Russian Foundation for Basic Research financial support within the framework of the research project No. 14-45-01044 "r_yug_a”. See results of the experiments at http://blacksea-model.ru

\section{REFERENCES}

1. Pavlushin, A.A., Shapiro, N.B., Mikhaylova, E.N. \& Korotaev, G.K., 2015, "Two-layer eddyresolving model of wind currents in the Black Sea”, Physical oceanography, no. 5, pp. 3-12.

2. Holland, W.R., Lin, L.B., 1975, "On the generation of mesoscale eddies and their contribution to the oceanic general circulation. I. A preliminary numerical experiment. II. A parameter study", J. Phys. Oceanogr., iss. 5, no. 4, pp. 642-657.

3. Migulin, V.V., Medvedev, V.I. \& Mustel', E.R. [et al.], 1988, “Osnovy teorii kolebaniy [Oscillation theory fundamentals], Moscow, Nauka, 391 p. (in Russian).

4. Ivanov, V.A., Belokopytov, V.N., 2011, “Okeanografiya Chernogo morya [Oceanography of the Black Sea]”, Sevastopol, MGI NAN Ukrainy, 212 p. (in Russian).

5. Ponomarev, V.I., Yurasov, G.I., 1994, “The Tartar (Mamiya) strait currents”, J. Kor. Soc. Coas. Ocean Engineers, vol. 6, no. 4, pp. 335-339.

6. Ponomarev, V.I., Fayman, P.A. \& Mashkina, I.V. [et al.], 2015, "Modelirovanie raznomasshtabnoy tsirkulyatsii $v$ severo-zapadnoy chasti Yaponskogo morya [Modeling of the large-scale circulation in the northwestern part of the Sea of Japan], Sistemy kontrolya okruzhayushchey sredy, Sevastopol, IPTS, iss. 2(22), pp. 65-73 (in Russian). 